\title{
Malnutrition and its associated factors among children under 5 years old in Putrajaya: a study protocol
}

\author{
Mohamad Hasnan Ahmad ${ }^{1 *}$, Nor Azian Mohd Zaki ${ }^{1}$, Fatimah Othman ${ }^{1}$, Azli \\ Baharudin $^{1}$, Ruhaya Salleh ${ }^{1}$, Cheong Siew Man ${ }^{1}$, Adibah Huda Mohd Zainul \\ Arifien ${ }^{1}$, Nurin Iman Ahmad Kamal', Noor Ani Ahmad ${ }^{1}$, Hazizi Abu Saad ${ }^{2}$, Poh \\ Bee Koon ${ }^{3}$, Mohd Azahadi Omar ${ }^{1,4}$ \& Tahir Aris ${ }^{1,5}$
}

${ }^{1}$ Institute for Public Health, National Institutes of Health, Ministry of Health Malaysia, Jalan Setia Murni U13/52, Seksyen U13, 40170 Shah Alam, Selangor, Malaysia; ${ }^{2}$ Faculty of Medicine and Health Sciences, Universiti Putra Malaysia, 43400 UPM Serdang, Selangor, Malaysia; ${ }^{3}$ Faculty of Health Sciences, Universiti Kebangsaan Malaysia, Jalan Raja Muda Abdul Aziz, 50300 Kuala Lumpur, Malaysia; ${ }^{4}$ Sector of Biostatistics and Data Repository, National Institutes of Health, Ministry of Health Malaysia, Jalan Setia Murni U13/52, Seksyen U13, 40170 Shah Alam, Selangor, Malaysia; ${ }^{\mathbf{5}}$ Institute for Medical Research, National Institutes of Health, Ministry of Health Malaysia, Jalan Setia Murni U13/52, Seksyen U13, 40170 Shah Alam, Selangor, Malaysia

\begin{abstract}
Introduction: Prevalence of malnourished children in Putrajaya was unexpectedly high in 2016. This paper describes the study protocol for a case-control study conducted to identify the associated factors of malnutrition among children under 5 years old in Putrajaya. Methods: This study involved two phases. Phase I was 'screening' where all children aged 6-59 months in 118 preschools and four government health clinics were measured for their weight and height. The World Health Organization Anthro software was used to determine the nutritional status of these children. Phase II was the 'interview' where children from screening were sampled into four pairs of case and control. The optimum sample size for the case of stunted, wasted, underweight, and overweight were $380,335,318$, and 308 , respectively. The same number of controls were recruited. Parents/caregivers of selected children were approached to obtain data on parental factors, children factors, food intake factors, and environmental factors that contributed to malnutrition. Data analysis was performed by multiple logistic regression in SPSS version 26. Results: Screening phase successfully measured 8,261 (50.1\%) children from an estimated 16,500 children under 5 years old in Putrajaya. The number of children who were stunted, wasted, underweight, and overweight were 2,105 (25.5\%), 512 $(6.2 \%), 1,516(18.4 \%)$, and $248(3.0 \%)$, respectively. As overweight was undersampled, the number of controls for overweight was doubled to increase the power of the study. Parents/caregivers of selected cases and controls were interviewed in their household or any other venues at their convenience. Conclusion: This protocol promises beneficial outputs to stakeholders and policy makers that can be used for combating malnutrition in children.
\end{abstract}

Keywords: Malnourished children, children under five, study protocol, case-control, Putrajaya

\footnotetext{
*Corresponding author: Mohamad Hasnan Ahmad

Institute for Public Health, National Institutes of Health, Ministry of Health Malaysia,

Jalan Setia Murni U13/52, Seksyen U13, 40170 Shah Alam, Selangor, Malaysia

Tel: (6)03-33628731; Fax: (6)(03)33627801; E-mail: hasnan.ahmad@moh.gov.my

doi: https://doi.org/10.31246/mjn-2020-0075
} 


\section{INTRODUCTION}

Malnutrition is a state of disordered nutrition, in which a combination of varying degrees of over- or undernutrition and inflammatory activity has led to a change in body composition, diminished functions and health outcomes (Soeters et al., 2017). Undernutrition is defined as inadequate or imbalanced intake or absorption of micro- or macronutrients that leads to multiple conditions including acute and chronic malnutrition and micronutrient deficiencies; while overnutrition usually is associated with overweight and obesity, a form of malnutrition where the amount of nutrients consumed exceeds the amount required for normal growth, development, and metabolism (Black et al., 2016). The co-existence of underand overnutrition in a population is recognised as a "nutrition transition", which has been linked to rapid economic development, urbanisation, and overall modernisation in both low- and middleincome countries (Shetty, 2013).

According to the findings from the National Health and Morbidity Survey (NHMS) 2016, the prevalence of stunting, wasting, underweight, and overweight among children under 5 years old in Malaysia were $20.7 \%, 11.5 \%, 13.7 \%$, and 6.4\%, respectively (IPH, 2016a). Compared to previous NHMS 2011 and NHMS 2015, the prevalences of stunting and underweight have increased throughout this period. However, for wasting, the prevalence decreased from NHMS 2011 to NHMS 2015, and increased in NHMS 2016. On the contrary, the prevalence of overweight increased from NHMS 2011 to NHMS 2015, but decreased in NHMS 2016 (IPH, 2015; IPH, 2011). The current categorisation of prevalence threshold for malnutrition in children under 5 years old discloses that stunting and wasting in Malaysia are of high public health significance, while overweight is regarded as medium public health significance. This categorisation predicts a huge burden on health care services in future if the current condition remains as high public health significance (De Onis et al., 2019).

Putrajaya, which is the administrative capital of Malaysia, recorded the fourth highest prevalence for stunting $(24.3 \%)$ among children under 5 years old in the country in 2016. Meanwhile, the prevalences for other malnutrition indicators - wasting (8.7\%), underweight $(12.9 \%)$, and overweight $(4.5 \%)$ in Putrajaya were approximately the same as the national prevalences (IPH, 2016a). These malnutrition issues among children under 5 years old, specifically in Putrajaya, has been highlighted in the government's cabinet meeting. The top management has since requested for more data and immediate action. One of the proposed efforts is a comprehensive study to find out the factors contributing to childhood malnutrition in Putrajaya.

Table 1 illustrates the associated factors of malnutrition in children according to previous local and international studies. Generally, four categories of associated factors can be formed, which are parental factors, children factors, food intake factors, and environmental factors (Stewart et al., 2013; Pasricha \& Biggs, 2010; Wong, Moy \& Nair, 2014; Harding, Aguayo \& Webb, 2018; Rahman, 2016; Andegiorgish et al., 2012; Park et al., 2013). All associated factors in Table 1 were translated into assessment modules in this study. This paper describes the study protocol of a casecontrol study to identify the associated factors of malnutrition among children under 5 years old in Putrajaya. 
Table 1. Associated factors of malnutrition among children under 5 years old from literature review

\begin{tabular}{|c|c|c|c|c|}
\hline Associated factors from previous studies & Stunted & Wasted & Underweight & Overweight \\
\hline \multicolumn{5}{|l|}{ Parental factor } \\
\hline Parental height & $\checkmark^{\text {adf }}$ & $\checkmark^{\mathrm{g}}$ & & \\
\hline Parental body weight status & & $\checkmark^{g}$ & & $\checkmark$ bc \\
\hline Lower education & $\checkmark$ adf & $\checkmark$ adf & $\checkmark$ adf & $\checkmark$ bc \\
\hline Working mother & & & & $\checkmark^{c}$ \\
\hline Low household income & $\checkmark$ adf & $\checkmark$ adfg & $\checkmark$ adf & \\
\hline Higher household income & & & & $\checkmark^{c}$ \\
\hline Pre-pregnancy BMI $>25 \mathrm{~kg} / \mathrm{m}^{2}$ & & & & $\checkmark$ bc \\
\hline Gestational diabetes mellitus & & & & $\checkmark$ bc \\
\hline \multicolumn{5}{|l|}{ Children factor } \\
\hline Low birth weight & $\checkmark$ adef & $\checkmark^{\text {def }}$ & $\checkmark$ adef & \\
\hline High birth weight & & & & $\checkmark$ bc \\
\hline Male in gender & $\checkmark^{\mathrm{f}}$ & $\checkmark^{\mathrm{fg}}$ & & $\checkmark$ bc \\
\hline Delayed initiation & $\checkmark$ df & & & \\
\hline More than four siblings & $\checkmark$ adef & $\checkmark$ ef & $\checkmark$ adef & \\
\hline Frequent illness (monthly) & $\checkmark$ adef & $\checkmark$ adef & $\checkmark$ adef & \\
\hline Worm infection & $\checkmark$ adef & $\checkmark$ adef & $\checkmark$ adef & \\
\hline Anaemia & & $\checkmark$ adf & $\checkmark$ adf & \\
\hline Rapid weight gain under 1 year & & & & $\checkmark^{c}$ \\
\hline Firstborn in the family & & & & $\checkmark^{c}$ \\
\hline \multicolumn{5}{|l|}{ Food intake factor } \\
\hline Non-exclusive breastfeeding & $\checkmark$ df & $\checkmark$ df & $\checkmark$ df & \\
\hline Early cessation of breastfeeding & $\checkmark$ df & $\checkmark$ df & $\checkmark \mathrm{df}$ & $\checkmark^{c}$ \\
\hline Complementary feeding not at 6 months & $\checkmark$ df & $\checkmark$ df & $\checkmark$ cf & $\checkmark^{c}$ \\
\hline Received bottle-feeding & & $\checkmark$ df & $\checkmark \mathrm{df}$ & $\checkmark \mathrm{c}$ \\
\hline Using pacifier & & $\checkmark$ df & $\checkmark$ df & \\
\hline Infrequent $\&$ inadequate feeding & $\checkmark$ adef & $\checkmark$ def & $\checkmark$ adef & \\
\hline High calorie and sugar intake & & & & $\checkmark^{\mathrm{c}}$ \\
\hline Low dietary diversity & $\checkmark$ adef & $\checkmark$ def & $\checkmark$ adef & $\checkmark^{c}$ \\
\hline Food insecurity & $\checkmark$ adef & $\checkmark$ ade & $\checkmark$ ade & \\
\hline \multicolumn{5}{|l|}{ Environmental factor } \\
\hline Inadequate child stimulation and activity & $\checkmark$ df & $\checkmark^{\mathrm{d}}$ & $\checkmark^{\mathrm{d}}$ & $\checkmark$ bc \\
\hline Poor care practice & $\checkmark \mathrm{df}$ & $\checkmark^{\mathrm{d}}$ & $\checkmark^{\mathrm{d}}$ & $\checkmark \mathrm{c}$ \\
\hline Screen time $>2$ hours & & & & $\checkmark c$ \\
\hline Long sleep duration & & & & $\checkmark^{c}$ \\
\hline
\end{tabular}

a Pasricha \& Biggs, 2010

${ }^{\mathrm{b}}$ Andegiorgish et al., 2012

${ }^{c}$ te Velde et al. 2012

d Stewart et al., 2013

${ }^{\mathrm{e}}$ Wong et al., 2014

${ }^{\mathrm{f}}$ Rahman, 2016

${ }^{\mathrm{g}}$ Harding et al., 2018 


\section{MATERIALS AND METHODS}

\section{Study design}

This was a case-control study to identify the associated factors of malnutrition among children under five in Putrajaya. Four case groups were formed, which were stunted [height-for-age <-2 standard deviations (SDs)], wasted [Body mass index (BMI)-for-age <-2SDs], underweight (weight-for-age <-2SDs), and overweight (BMI-for-age $>2$ SDs). The control group consisted of children who had normal $z$-scores for all malnutrition indicators (2SDs to -2SDs).

\section{Study location}

This study was conducted from September 2018 to January 2019 in Putrajaya. It involved two phases screening and interview. Screening was conducted in all 118 preschools and all four government health clinics in Putrajaya from 12 September 2018 to 12 October 2018. The interview phase with the selected participant's parents / caregivers was conducted from 16 October 2018 to 31 January 2019 in their house or office, their child's kindergarten or any other venues at their convenience.

\section{Study population}

Children aged 6 to 59 months old, Malaysia citizen, and living in Putrajaya for at least six months were the inclusion criteria for this study. The exclusion criteria were children who were mentally or physically disabled, ill at the time of data collection, and children with a chronic disease that caused them the inability to take part in this study.

\section{Sample size estimation}

The sample size of this study was calculated based on the objective of identifying the associated factors (refer to Table 1) of stunting, wasting, underweight, and overweight among children under 5 years old. Sample size calculation was done using PS (Power and Sample Size Calculation) software based on the formula for comparing two proportions, according to identified associated factors in the findings from NHMS 2016 and other previous studies, with type 1 error $(\alpha)$ equals to 0.05 and power $(\beta)$ equals to 0.80 . The minimum sample size for the groups of stunted, wasted, underweight, and overweight were 380, 335, 318, and 308, respectively. Each case group was compared with the same number of children with normal nutritional status. The ratio of case to control in this study was one to one.

\section{Respondent recruitment}

Respondent recruitment for phase I (screening) was carried out in all 118 registered preschools under the Department of Social Welfare and all four government health clinics in Putrajaya. All available children who came into these facilities during the screening period were included in the database.

Respondent recruitment for phase II (interview) began after the selection of children for case and control groups from the screening database. Database of the children from screening were divided into five groups (stunted, wasted, underweight, overweight, and normal). Random between function in Excel was used for random selection of 380 stunted children, 335 wasted children, 318 underweight children, and 308 overweight children. The selected cases from each group were then tabulated by sex and age groups (6 -11 months, 12-35 months, and 3659 months). Then, the control groups were recruited by matching the sex and age group of each case group using the random between function in Excel with a one to one ratio. Ethics approval for the study was obtained from the Medical Research Ethics Committee (MREC), 
Ministry of Health Malaysia (NMRR-18847-41455). Parents or caregivers of selected participants signed the consent forms prior to taking measurements and participating in the interview sessions.

\section{Tools and instrument}

Four sections of data collection were carried out. The first was a face-to-face interview with parents or caregivers by trained interviewers. The collected information were saved in tablets using a custom designed application, named Survey Creating System (SCS). The information included sociodemographic and socioeconomic characteristics, participants and mothers' health and medical history, knowledge and practice of parents or caregivers towards child feeding, dietary behaviour of the child, infant and young child feeding (IYCF) history (WHO, 2010), food security (Radimer/Cornell hunger and food security instrument (Sharif \& Ang, 2001), and early years physical activity questionnaire (EY-PAQ) (Bingham, 2016).

The second section, which was anthropometric measurements for children and their parents or caregivers, were measured by nurses. Body weight was measured using the TANITA Personal scale HD 319 (TANITA Cooperation, Tokyo, Japan) and height was measured using a SECA Stadiometer 213 (SECA Cooperation, Humberg, German). The value of the measurement was rounded to the nearest $0.1 \mathrm{~kg}$ for weight and $0.1 \mathrm{~cm}$ for height. For baby or children who could not stand properly, their weight was measured using the SECA 354 digital baby scale and length using the SECA 210 mobile baby measuring mat. The World Health Organization (WHO) Anthro Software version 3.2.2 was used to identify eligible cases and controls (WHO, 2011a).

The third section was an anaemia test. Since anaemia is a risk factor for undernutrition in children, this study only measured haemoglobin levels of stunted, wasted, and underweight children, as well as their respective controls (Pasricha \& Biggs, 2010; Khan, Awan \& Misu, 2016). Haemoglobin levels of children were tested from a fingerprick blood sample using the portable HemoCue ${ }^{\circledR}$ Haemoglobin 201 analyser (HemoCue AB, Ängelholm, Sweden). Anaemia in children is defined as a haemoglobin concentration of less than $11.0 \mathrm{~g} / \mathrm{dl}$ as suggested by WHO (WHO, 2011b).

The fourth section was collecting data from a three-day food diary. The three-day food diary (two days weekdays and one day weekend) of children was completed by their parents or caregivers. For children who went to preschools, their teachers were given the responsibility to record their food intake in the food diary during weekdays. The information provided were verified by the interviewers before data entry. Total energy and nutrient intakes were analysed using the NutritionistPro software.

Field preparation and logistic support Data collection was carried out by six teams. Each team consisted of a team leader, a research assistant, and a nurse. The list of preschools in Putrajaya was obtained from the Department of Social Welfare. All 118 preschools were informed about this study by the Department of Social Welfare. Invitation letters to participate were distributed to parents or caregivers by the preschools' management staffs.

Body weight and height of preschool children were measured during the screening phase. Information of child's name, date of birth, gender, name of parents or caregivers, living address, and contact number of parents or caregivers were obtained from the preschool authorities. In addition, 


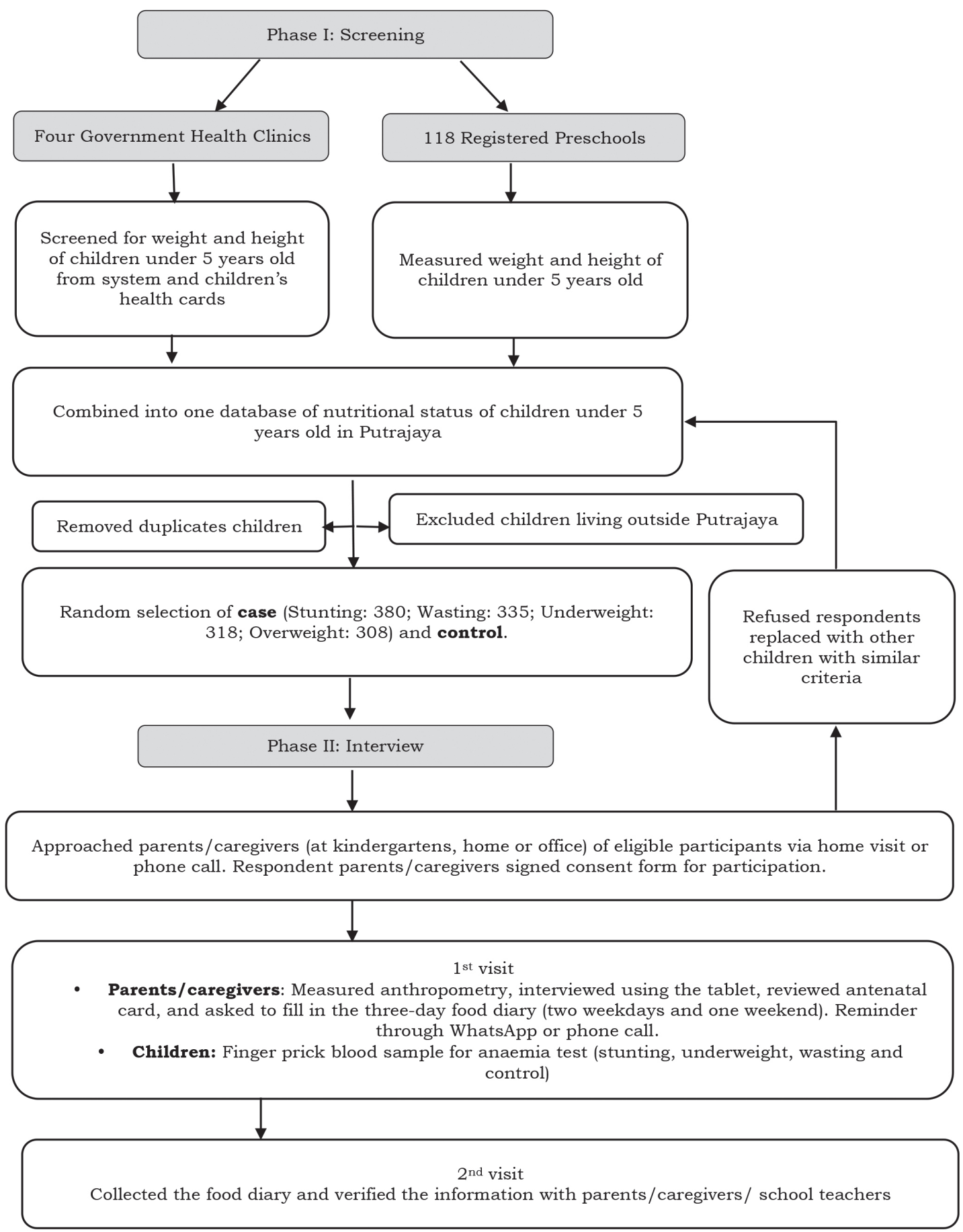

Figure 1. Data collection flow from screening phase to interview phase 
the data collectors visited all four government health clinics in Putrajaya, located in Precincts 9, 11, 14, and 18. Information on the latest weight and height, name of child, date of birth, gender, name of parents or caregivers, living address, and contact number of parents or caregivers were obtained from the patients' management system or record books at the clinics.

Data obtained from all 118 preschools and four government health clinics were combined in one database. Duplicates of children from preschools and government health clinics were removed, while children living outside of Putrajaya were excluded from the database. Children who met the inclusion criteria were divided into five groups, which were stunted, wasted, underweight, overweight, and normal. Then, they were randomly selected according to the sample size required for each group.

During the interview phase, parents or caregivers of the selected children were approached through phone calls and invitation letters. The data collectors visited all parents or caregivers who agreed to participate in this study. Two health clinics were established as interview centres to provide additional option for parents or caregivers. Figure 1 demonstrates the data collection flow from screening phase to interview phase.

\section{Data management}

Each selected respondent was given a unique identification number (ID) prior to the interview phase. Data from the tablets were sent directly to the server of the Institute for Public Health (IPH) via internet. Data processing and management were conducted simultaneously by the data manager at IPH to ensure that all data were correctly received. Data were then extracted into the Microsoft Excel format. The three-day food diary were delivered to IPH weekly. The data entry team in IPH received and converted the participants' food intakes into gram based on standard recipes. After conversion to gram, the data were keyed into the NutritionistProTM software version 7.5 (Axxya System, Washington, United States of America). NutritionistProTM analysed the food intakes into calories and nutrient intakes by referencing the Malaysian Food Composition Database (MyFCD) and other established databases.

\section{Data analysis}

Descriptive statistics were used to describe the studied variables. Single and multiple logistic regression were performed to analyse potential factors of malnutrition such as the questionnaire on maternal and birth characteristics. Odds ratio $(O R)$ and its $95 \%$ confidence interval (CI) were estimated and computed for each significant factor and continuous data using binary logistic regression. A factor with an $O R$ significantly $(p<0.05)>1.00$ was taken as a risk factor of child malnutrition, while an $O R$ significantly $(p<0.05)<1.00$ was regarded as a protective factor. The goodness of fit for the final logistic regression model was analysed using the Hosmer-Lemeshow technique. The statistical significant level was set at 0.05. All statistical analyses were performed using SPSS version 26.

\section{RESULTS}

Out of 8,261 children under 5 years old recruited during screening, a total of $2,105(25.5 \%), 512(6.2 \%), \quad 1,516$ $(18.4 \%)$, and $248(3.0 \%)$ were found to be stunted, wasted, underweight, and overweight, respectively (Table 2). There were children who were found to have $>1$ indicator of malnutrition. Therefore, they were allocated into $>1$ case group. 
Table 2. Distribution of case groups (stunted, wasted, underweight, and overweight) and control (normal) from this study and findings from NHMS 2016

\begin{tabular}{lcccc}
\hline \multirow{2}{*}{ Indicator } & \multicolumn{3}{c}{ Screening Phase } & NHMS 2016 \\
\cline { 2 - 5 } & $\begin{array}{c}\text { Sample size } \\
\text { required }\end{array}$ & $n$ & $\%(95 \%$ CI) & $\%(95 \%$ CI) \\
\hline Stunted & 380 & 2105 & $25.5(24.57,26.43)$ & $24.3(19.05,30.42)$ \\
Wasted & 335 & 512 & $6.2(5.68,6.72)$ & $8.7(6.39,11.75)$ \\
Underweight & 318 & 1516 & $18.4(17.56,19.24)$ & $12.9(9.56,16.76)$ \\
Overweight & 308 & $248^{+}$ & $3.0(2.63,3.37)$ & $4.5(3.18,6.36)$ \\
Normal & 380 & 5223 & $63.2(62.22,63.22)$ & - \\
\hline
\end{tabular}

${ }^{\dagger}$ less than required sample size

In general, the number of overweight children recruited into the overweight group was less than the minimum required sample size. A total of 5,223 $(64.0 \%)$ children were normal and eligible to be allocated into the control group.

Table 2 shows the results of screening for this study with comparison to the findings from NHMS 2016 in Putrajaya. On average, the prevalences of different malnutrition groups in this study were comparable with the results from NHMS 2016, except for underweight. The prevalence of underweight was significantly higher in this study according to $95 \%$ confident interval compared to NHMS 2016.

\section{DISCUSSION}

This paper described the methodology to identify or determine factors associated with malnutrition among children under five years old in Putrajaya. The casecontrol study design was chosen as this was the best approach to determine whether an exposure (tested variable) is associated with an outcome (case/ control) with limited time and minimum cost (Song \& Chung, 2010). The cases and controls were also matched by sex and age groups to improve the study efficiency by enhancing precision in the analysis. The increase in efficiency occurs because it ensures similar number of cases and controls in confounder strata (sex and age groups) (Pearce, 2016).

The ratio of one-to-one for case group and control group was applied in this study. At the same time, all four case groups were compared with the same control group in order to provide adequate power for this study with minimum sample size. It also shortens the data collection time and reduces study expenses. However, this study has a limitation where only 248 overweight children were found, which was lower than the minimum sample size required for the overweight group. Therefore, the number of participants in the control group was increased to double the ratio of overweight to control as one to two so that it can provide adequate power to detect a significant association (Kang, Choi \& Koh, 2009).

The phase I results from this study found that $25.5 \%$ of children under five years old in Putrajaya were stunted, 6.2\% were wasted, $18.4 \%$ were underweight, and $3.0 \%$ were overweight. Through $95 \%$ $\mathrm{CI}$, the prevalence of underweight was significantly higher when compared to the findings in NHMS 2016 for Putrajaya (12.9\%). Meanwhile, the prevalence for the rest of the malnutrition indicators in this study were more comparable with the findings for Putrajaya in NHMS $2016(24.3 \%$ stunted, $8.7 \%$ wasted, and $4.5 \%$ overweight) (IPH, 2016a). 
The significantly higher prevalence of underweight children in this study was mainly due to the sampling technique used. Compared to household visits in NHMS 2016, our data collection was conducted in preschools and government health clinics in Putrajaya. Based on the current practice of 'Standard Operation Procedure for Malnourished Children' (MOH, 2015), underweight children are required to come to health clinics for regular monitoring of their weight status. Therefore, the bulk of underweight children sampled from health clinics has influenced our data, increasing its prevalence.

The prevalence of malnutrition from this study or NHMS 2016 for Putrajaya was lower compared to the prevalence in urban areas of Indonesia. About $34.9 \%, 23.4 \%$, and $13.0 \%$ children from the urban areas of Indonesia were stunted, underweight, and overweight, respectively (Rachmi et al., 2016). Meanwhile, in the urban areas of the Philippines, $26.0 \%, 18.0 \%$, and $5.0 \%$ of their children were stunted, underweight, and wasted, respectively (Rohner et al., 2013). However, the current Global Nutrition Report 2020 found a significant urban-rural divide especially in stunting prevalence. Out of 110 countries with available stunting data by location, children living in rural areas (35.6\%) had higher stunting rates than those living in urban areas (25.6\%). However, prevalences of wasting and overweight were more comparable. There were $12.4 \%$ wasted children reported in rural versus $11.4 \%$ in urban areas, and $4.0 \%$ overweight children in rural versus $4.9 \%$ in urban areas (Micha et al., 2020).

Although this screening phase successfully recruited $>50 \%$ of the children population in Putrajaya, the convenient selection of the participants from clusters (118 preschools and four government health clinics) was not comparable with the random selection of participants from NHMS 2016. NHMS 2016 used a complex sampling approach with a sample frame provided by the Registration Department (IPH, 2016).

The sociodemographic characteristics in this study were not evenly distributed as Putrajaya is densely populated by government servants, and majority are Malays (DOSM, 2017). Therefore, the findings cannot be generalised across different ethnicities. Nevertheless, we believe the outcomes of this study will provide sufficient information for stakeholders and policy makers to plan an effective prevention to reduce the prevalence of malnutrition among children in Putrajaya or other locations with similar characteristics.

\section{CONCLUSION}

This case-control study was planned to identify the associated factor of malnutrition among children in Putrajaya. Therefore, the results of this study provides valuable information to combat the issue of malnutrition among children under 5 years old in Putrajaya.

\section{Acknowledgement}

The authors would like to thank the DirectorGeneral of Health Malaysia for his permission to publish this manuscript. Sincere appreciation to all participants who have contributed their precious time and information to this survey; without them, there would not be a success to this study.

\section{Authors' contributions}

MHA, principal investigator, conceptualised and designed the study, led the data collection, prepared the draft of the manuscript and reviewed the manuscript; NAMZ, co-principal investigator and led the data collection; FO, conceptualised and designed the study and led the data collection; $\mathrm{AB}$, led the logistic support and data collection, reviewed the manuscript; RS, advised on data collection and interpretation, and reviewed the manuscript; CSM, led data management in central and reviewed the manuscript; AHMZA, managed data entry, prepared the draft and reviewed the manuscript; NIAK, managed data entry, prepared the draft and reviewed the manuscript; NAA, conceptualised and designed the study, advised in analysis and reviewed the manuscript; HAS, 
advised in analysis and reviewed the manuscript and interpretation; PBK, advised in data collection, assisted in drafting of the manuscript, reviewed the manuscript; MAO, advised in data collection and data analysis, and reviewed the manuscript; TA, conceptualised and designed the study, advised in data collection and data analysis, and reviewed the manuscript.

\section{Conflict of interest}

There is no conflict of interest.

\section{References}

Andegiorgish AK, Wang J, Zhang X, Liu X \& Zhu H (2012). Prevalence of overweight, obesity, and associated risk factors among school children and adolescents in Tianjin, China. Eur $J$ Pediatr 171(4):697-703.

Bingham DD, Collings PJ, Clemes SA, Costa S, Santorelli G, Griffiths P \& Barber SE (2016). Reliability and validity of the early year's physical activity questionnaire (EY-PAQ). Sports 4(2):30.

Black RE, Levin C, Walker N, Chou D, Liu L, Temmerman M \& Group DRA (2016). Reproductive, maternal, newborn, and child health: key messages from disease control priorities 3rd edition. The Lancet 388(10061):2811-2824.

De Onis M, Borghi E, Arimond M, Webb P, Croft T, Saha K, De-Regil LM, Thuita F, Heidkamp R, Krasevec J \& Hayashi C (2019). Prevalence thresholds for wasting, overweight and stunting in children under 5 years. Public Health Nutr 22(1):175-9.

DOSM (2017). Putrajaya Population Table 2017. Department of Statistic Malaysia. From https://www.dosm.gov.my/v1/ index.php? r = colum n / cone \& m en u id=bkJnUlk2WXUyTOhVWm5IZXlubERjUT09 [Retrieved May 12 2018]

Harding KL, Aguayo VM \& Webb P (2018). Factors associated with wasting among children under five years old in South Asia: Implications for action. PloS One 13(7):e0198749. doi:10.1371/ journal.pone.0198749.

IPH (2011). National Health and Morbidity Survey 2011 (NHMS 2011). Vol. II: Non-Communicable Diseases. Institute for Public Health, Bangsar, Kuala Lumpur.

IPH (2015). National Health and Morbidity Survey (NHMS) 2015. Non-Communicable Disease: Volume II: Non-communicable disease, risk factors and other health problems. Institute for Public Health, Bangsar, Kuala Lumpur.
IPH (2016a). National Health and Morbidity Survey (NHMS) 2016. Maternal and Child Health: Volume II: Maternal and Child Health Findings. Institute for Public Health, Bangsar, Kuala Lumpur

IPH (2016b). National Health and Morbidity Survey (NHMS 2016): Maternal and Child Health. Volume I: Methodology and General Findings. Institute for Public Health, Bangsar, Kuala Lumpur

Kang MS, Choi SH \& Koh IS (2009). The effect of increasing control-to-case ratio on statistical power in a simulated case-control snp association study. Genomics Inform 7(3):14851.

Khan JR, Awan N, \& Misu F (2016). Determinants of anemia among 6-59 months aged children in Bangladesh: evidence from nationally representative data. BMC pediatrics 16(1):3.

Micha R, Mannar V, Afshin A, Allemandi L, Baker P \& Battersby J (2020). Global nutrition report: action on equity to end malnutrition 2020 Technical Report. Development Initiatives, Bristol, UK.

MOH Malaysia (2015). Garis Panduan Program Pemulihan Kanak-Kanak Kekurangan Zat Makanan. Bahagian Pemakanan, Kementerian Kesihatan Malaysia 2015. Ministry of Health Malaysia, Putrajaya.

Park MH, Sovio U, Viner RM, Hardy RJ \& Kinra S (2013). Overweight in childhood, adolescence and adulthood and cardiovascular risk in later life: pooled analysis of three british birth cohorts. PloS one 8(7):p.e70684.

Pasricha SR \& Biggs BA (2010). Undernutrition among children in South and South-East Asia. $J$ Paediatr Child Health 46(9):497-503.

Pearce N (2016). Analysis of matched case-control studies. BMJ 352:1969.

Rahman A (2016). Significant risk factors for childhood malnutrition: evidence from an Asian developing country. Sci J Public Health 4(1-1):16-27.

Rachmi CN, Agho KE, Li M, \& Baur LA. (2016). Stunting, underweight and overweight in children aged 2.0-4.9 years in Indonesia: prevalence trends and associated risk factors. PloS one 11(5):e0154756.

Rohner F, Bradley AW, Grant JA, Elizabeth AY, Lebanan MAO, Rayco-Solon P \& Saniel OP (2013). Infant and young child feeding practices in urban Philippines and their associations with stunting, anemia, and deficiencies of iron and vitamin A. Food and Nutrition Bulletin 34(1):17-34. 
Sharif ZM \& Ang M (2001) Assessment of food insecurity among low income households in Kuala Lumpur using the Radimer/Cornell food insecurity instrument-a validation study. Mal $J$ Nutr 7(1):15-32.

Shetty P (2013). Nutrition transition and its health outcomes. Indian J Pediatr 80(1):21-27.

Soeters P, Bozzetti F, Cynober L, Forbes A, Shenkin A \& Sobotka L (2017). Defining malnutrition: a plea to rethink. Clin Nutri 36(3):896-901.

Song JW \& Chung KC (2010). Observational studies: cohort and case-control studies. Plast Reconstr Surg 126(6):2234.

Stewart CP, Iannotti L, Dewey KG, Michaelsen KF \& Onyango AW (2013). Contextualising complementary feeding in a broader framework for stunting prevention. Matern Child Nutr 9:27-45.

te Velde SJ, Van Nassau F, Uijtdewilligen L, Van Stralen MM, Cardon G, De Craemer M \& ToyBox-study group. (2012). Energy balancerelated behaviours associated with overweight and obesity in preschool children: a systematic review of prospective studies. Obes Rev 13:5674.
Wong HJ, Moy FM \& Nair S (2014). Risk factors of malnutrition among preschool children in Terengganu, Malaysia: a case control study. BMC Public Health 14(1):785.

WHO (2010). Indicators for assessing infant and young child feeding practices: part 2: measurement 2010. World Health Organization, Geneva.

WHO (2011a). WHO Anthro (version 3.2.2, January 2011) and macros. World Health Organization, Geneva, Switzerland. From http://www. who. int/childgrowth/software/en. 2011. Retrieved August 24, 2018.

WHO (2011b). Haemoglobin concentrations for the diagnosis of anaemia and assessment of severity (No. WHO/NMH/NHD/MNM/11.1). World Health Organization, Geneva. 